\title{
NEED OF STUDY AT NATIONAL SCALE
}

I continue to read the JNMA with interest.

There was a letter in Vol. 40 (140), Oct.-Dec. 2001 which interested me, but I have not seen any comment since.

"Need of study at National Scale." It is worth encouraging.

Perhaps Dr. Paudel as a colleague could present a paper at a conference bringing the value of such a study.

And in the early stages, keep it simple and non threatening - responding to such unspoken questions as "what's in it for me ? - cost - time \& trouble" etc.

Perhaps keep it to incidence, include private as well as non private hospitals \& provide feed back.

This is by no means a comprehensive letter, may be a caricature of one and intended primarily as an encouragement.

Frank Garlick

27 Chartwell ST

Aspley QLD 4034

Australia

(Dr. Garlick is the Former Medical

Superintendent of Patan Hospital)

\section{Request to the Readers}
All the readers are encouraged to send the feedback for publication in "Letter to the Editor" section.

JNMA Editorial board welcomes general and specific comments, criticisms which will ultimately guide us to improve the quality of the journal. 


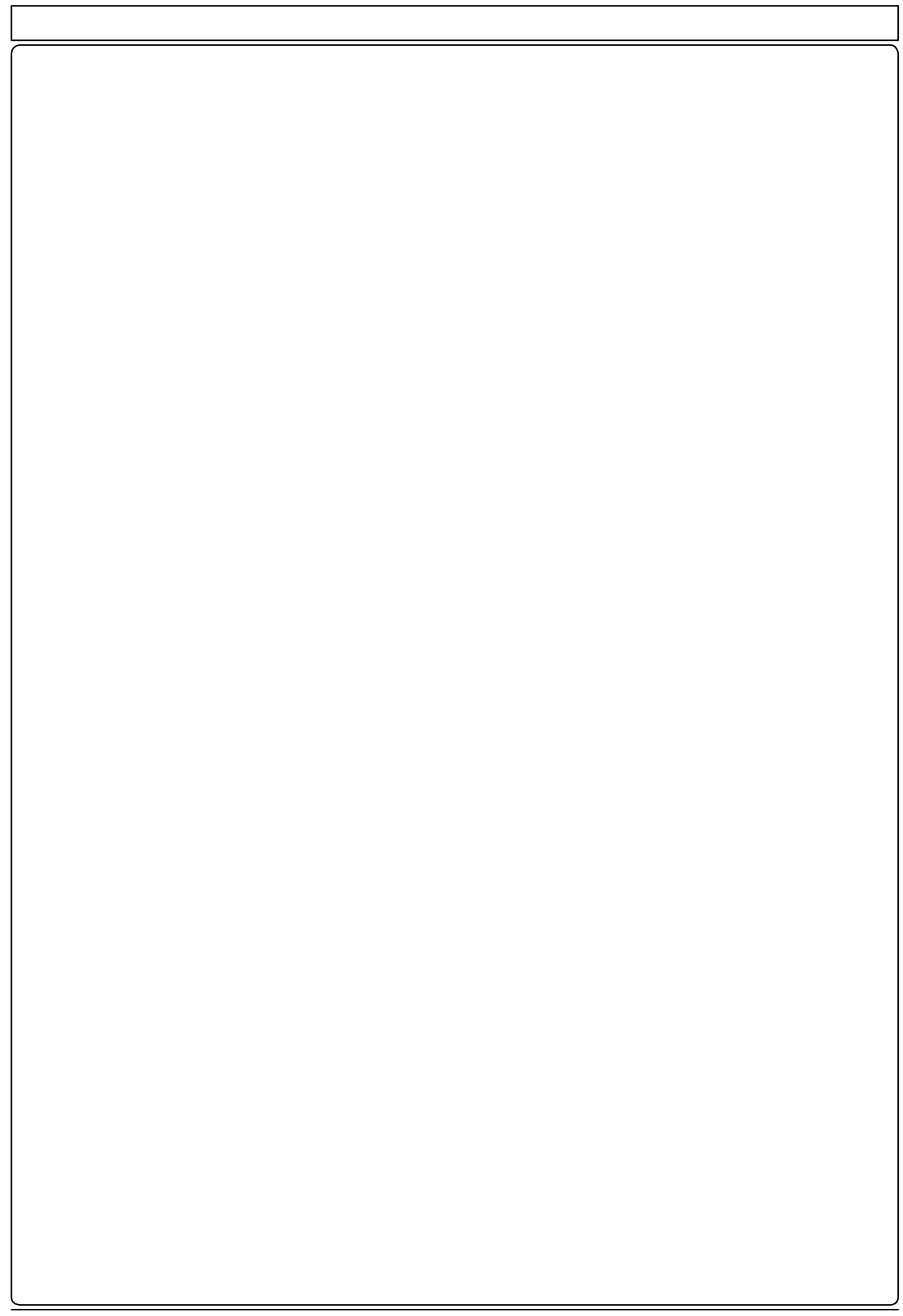

JNMA, July - September, 2002, 41 


$$
\begin{aligned}
& \text { Z y d u s } \\
& \text { c a di } 1 \text { a }
\end{aligned}
$$

Zydus Cadila's Class Products

Dexona 0.5 mg Tab. / 2 ml Inj.

Ciprobid Tab. 250 / 500 mg

Depin $5 \mathrm{mg} / 10 \mathrm{mg} / 20 \mathrm{mg}$ Retard

Ocid Cap. 10 / 20 mg

Oflin Tab. 100 / 200 / 400 mg. O.D.

Cadila Health Care Itd.

Zydus Tower

Satellite Cross Roads,

Ahmedabad - 380015

India 


\title{
With Best Compliments
}

\author{
From
}

OZONE Pharmaceuticals Itd.

The Makers of

- The most advanced nitric oxide donor OSIL - 500 MG Capsule

- Cynocal - Tab. \& Inj.

- Nubex - Forte Capsule

- Zerox Tab. 150 Mg \& Suspension 60 MI

- $\quad$ FAM - H2

- Topex - BR

$\&$

- D. F. O. Gel. - Analgesic Cream 
It is customary for $\mathrm{HO} / \mathrm{SHO}$ position at $\mathrm{KMC}$ Teaching Hospital to start on 1st Shrawan \& 1st Magh B.S. every year. Interviews for positions will be held about 15 days prior to this viz 1st Jan \& 1st July A.D. Applications should state:

1 Postappli eff or (HO/SHO). Tuockpar trental pr ef er ences nterisf pri ori ty

2 Hotocopi esf acaderinc certi fi catefrorbLC.

3 Certi fi cate of regtrati onbyNMC.

4 Certi fi cateregra ngrei ouposs f any

For further particulars contact:

Personnel Manager,

KMC Teaching Hospital

Sinamangal, Kathmandu.

Phone : 477920 .

Email: Kmc@unlimit.com

NB: Doctors with postgraduate qualifications, who are interested in joining Kathmandu Medical College, should enquire regarding existing vacancies. 


\section{Ue Fentily Congratulate}

Journal of the Nepal Medical Association (JNMA)

On the publication

of their quarterly issue

From the Makers of:

Welmox 250/500mg Caps and Welmox 125/250 DT

(Amoxycillin)

Welclox

(Amoxy $250+$ Cloxa 250mg)

Salmol $4 \mathrm{mg}$

(Salbutamol 4mg)

and

Tinizol 300/500/1000mg

(Tinidazole)

Everest Pharmaceuticals (Pvt.) Itd.

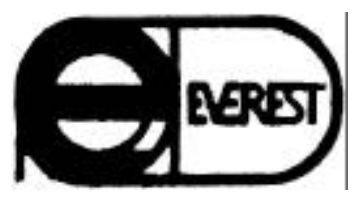

P.O. Box: 3695, Kathmandu, Nepal.

Tel: 977-1-259959, Fax: 977-1-260783

Email: epl@wlink.com.np 
Best Wishes for a

Grand Success

of the

20 th All Nepal Medical Conference of the

NEPAL MEDICAL ASSOCIATION

German Remedies Limited Head Office: P.O. Box: 8570, Mumbai 400018 Tel.: 4935528. Fax: 4950327

C/0. Medi Sales, Gha-2-485, Durbar Marg, Post Box: 1709, Kathmandu, Nepal. Tel.: (01) 224634, 222771 
With

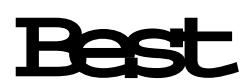

Campliments

\section{From:}

NATIONAL HEALTHCARE 
With Best Compliments

\section{From \\ YETICHEM GROUP}

44-Mahadev Marg, Bag Durbar, Sundhara, Kathmandu Phone No.: 243356, 225244, Fax No.: (1) 228244

E-mail: yetichem@mos.com.np

(Specialised in Anti Cancer \& Life Saving Medicines)

\section{Super Distributors for Nepal}

Cipla Ltd., Mumbai

Rhone-Poulenc (India) Ltd.

Hoechst Marion Roussel Ltd.

Neon Laboratories Ltd., Mumbai

Dabur India Ltd., Pharmaceuticals Divs. Shantha Biotecnics Pvt. Ltd., Hyderabad Jayson Pharmaceuticals Ltd., Bangladesh F. Hoffmann-La Roche Ltd., Switzerland 